\title{
Obesity and Surgical Treatment
}

\section{A Cost-Effectiveness Assessment for Sweden}

\author{
SIXTEN BORG ${ }^{1,2, *}$, INGMAR NÄSLUND ${ }^{3}$, \\ ULF PERSSON $^{1}$, KNUT ÖDEGAARD ${ }^{1}$ \\ ${ }^{1}$ The Swedish Institute for Health Economics (IHE), Lund, Sweden. \\ ${ }^{2}$ Health Economics Unit, Department of Clinical Science in Malmö, Lund University, Lund, Sweden. \\ ${ }^{3}$ University Hospital Örebro, Surgical department, Örebro, Sweden.
}

\begin{abstract}
The rising trend in the prevalence of obesity has become a major public health concern in many countries during the past decades, partly because being obese is associated with comorbidities and death. The cost of treatment for obesity related diseases has become a heavy burden on the national health care budget in many countries. The treatment options for obesity are mainly weight management therapies in the form of diet and exercise, pharmacological therapy, and surgery. The incidence of bariatric surgery in Sweden has increased eight-fold in the last decade. Our objective was to assess the cost-effectiveness of gastric bypass surgical treatments for obesity in adult patients, in comparison with conventional treatment, in Sweden from a societal perspective. The conventional treatment alternative consisted of the prevalent mixture of non-surgical obesity treatments. A model of individual patients was used to simulate the outcomes of the patients in terms of treatment costs, indirect costs, life years, and quality adjusted life years (QALY) over a lifetime perspective. In patients with a Body Mass Index of 40-44 $\mathrm{kg} / \mathrm{m}^{2}$, surgery was estimated to be cost-saving in men and judged cost-effective in women, with an incremental cost per QALY gained of SEK 26 thousand (EUR 3 thousand). The incremental cost associated with gastric bypass decreases with BMI, increases with the patient's age, and is higher in women than in men. Taking patient characteristics and uncertainty in input data and model design into account, the incremental cost is estimated to be at most SEK 160 thousand per QALY gained (about EUR 18 thousand per QALY). In conclusion, gastric bypass surgery appears to be a cost-effective intervention compared to conventional treatment in adult persons with obesity in Sweden.
\end{abstract}

Key words: obesity, bariatric surgery, health economic model, cost-effectiveness analysis, QALY

\section{Introduction}

Obesity is the state of excess body fat relative to lean body mass. The rising trend in the prevalence of obesity has become a major public health concern in many countries during the past decades. This is partly because being obese is associated with a greatly elevated risk of contracting a range of different diseases such as type 2 diabetes, heart disease, hypertension and stroke, hyperlipidaemia, osteoarthritis, sleep apnoea and certain types of cancer, and partly because the mortality risk is greatly elevated in people with obesity. Overweight and obesity are important risk factors for cardiovascular disease, which causes over 17 million deaths every year (WHO 2005). The World Health Organization estimates that one billion people are overweight or obese globally (WHO 2013). A frequently used

* Correspondence to: Sixten Borg, The Swedish Institute for Health Economics, Box 2127, SE-220 02, Lund, Sweden, Phone +46 463291 00, Fax +46 461216 04, E-mail: sb@,ihe.se 
marker for obesity is the Body Mass Index (BMI), calculated as the ratio between a person's weight in $\mathrm{kg}$ and the square of the person's length in meter. BMI is used to classify persons as underweight (BMI $<18.5 \mathrm{~kg} / \mathrm{m} 2)$, with a normal weight $(18.5 \leq \mathrm{BMI}<$ 25), as overweight $(25 \leq$ BMI $<30 \mathrm{~kg} / \mathrm{m} 2)$, and obese $($ BMI $\geq 30 \mathrm{~kg} / \mathrm{m} 2)$ (WHO/NUT/NCD/98 1997). Statistics from European countries shows that more than 50 per cent of the population can be classified as overweight and up to 30 per cent as clinically obese (Cremieux et al. 2008). In Sweden, 28\% of all Swedish women and 42\% of all men were overweight, and $11 \%$ of all women and $12 \%$ of all men were obese in 2011 (Statistics Sweden 2013a).

The cost of treatment for obesity related diseases has become a heavy burden for the national health care budget in many countries. One estimate from the US shows that 9.1 per cent of total medical spending there could be attributed to overweight and obesity (Finkelstein et al. 2003). The cost burden is not as heavy in Europe as in the US, because the prevalence of obesity and overweight has not yet reached US levels (SBU 2002). In 2011, the total yearly costs of healthcare and drugs attributable to overweight and obesity in Sweden were estimated to about EUR 470 million (Persson and Ödegaard 2011).

The treatment options for obesity are mainly weight management therapies in the form of diet and exercise, pharmacological therapy, and surgery. Surgical treatment of obesity has existed for over 50 years and has gone from a practice endeavoured by a few clinicians to now being common and widespread. Today, surgical intervention is a common strategy in Sweden. The annual number of bariatric surgeries has increased from a few hundred in the late 1990s to a peak of about 8,000 (89 per 100000 inhabitants) in the period from 2010 to 2012 (Scandinavian Obesity Surgery Registry 2012). The costs for surgical treatment of obesity are estimated to about EUR 45 million in 2010 and 70 million in 2011, which should be set in relation to the population size of 9 million in Sweden (Persson and Ödegaard 2011).

An important question is whether surgical intervention on obesity is worthwhile, or in which cases it is worthwhile. Our previous study of the budget impact of bariatric surgery in Sweden used a health economic model to show that the cost of surgery is partly offset by other healthcare cost savings in a 10-year time horizon (Borg et al. 2012). The lifetime costs and whether they can be judged acceptable in relation to the accomplished health benefits, however, remain unknown. Thus, to study the cost-effectiveness of surgical intervention in a Swedish setting was a natural extension of our previous work.

In a review of the cost-effectiveness literature on surgical intervention for obesity, Avenell et al (2004) identified six studies, three of which were from the US (Chua and Mendiola 1995; Martin et al. 1995; Nguyen et al. 2001), and there was one study from each of the following countries: UK (Clegg et al. 2002), the Netherlands (van Gemert et al. 1999), and Sweden (Sjostrom et al. 1995). All but one of the studies were found to contain weaknesses and only two of the studies offered an assessment of lifetime costs and benefits of surgical intervention using a modelling framework. No modelling framework was used in the study from Sweden. The study was based on early data from the Swedish Obesity Study, which started collecting data during the late 1980s and early 1990s. More recently, Padwal et al (2011) presented a review of clinical and economic assessments of bariatric surgery. Retrieving 70 articles for review, they came up with 13 articles that met the criteria for a systematic review of the economic evidence. The evidence indicated that surgery resulted in incremental cost-utility ratios in the region of US $\$ 1,000-40,000$ per quality adjusted life year compared with non-surgical interventions. However, Padwal et al found three major short-comings in the reviewed cost-effectiveness models: (1) short term observational data/randomized clinical trial data were used to model long term effect on weight reduction; (2) the weight of a patient was assumed to remain stable over time, 
and (3) comorbidities associated with obesity were commonly ignored. Our model does not have any of these three shortcomings.

Our objective of the paper was to use our model to assess the cost-effectiveness of gastric bypass surgical treatment for obesity in adult patients, in comparison with conventional treatment, in Sweden from a societal perspective. The conventional treatment alternative consisted of the prevalent mixture of non-surgical obesity treatments given.

\section{Methods}

In order to carry out a cost-effectiveness analysis, one needs to estimate the relevant types of costs and health benefits, over an adequate period of time. Often, there is no single study that covers all aspects necessary for the analysis, and usually the follow-up periods of available studies are too short to encompass the desired time frame. A common solution is to create a health economic model into which data from several sources are combined so that the relevant costs and health benefits are covered, and so that an adequate time frame is covered. This usually includes translation of intermediate outcomes measures, e.g. BMI, into the endpoints required for the cost-effectiveness analysis. By necessity, assumptions must be made, and the reader is encouraged to keep this in mind and decide whether they are sound.

Our model of a person eligible for gastric bypass surgery is a micro-simulation model that simulates the outcomes of individual patients in terms of costs, life years, and QALYs over a 50 year time period, in order to attain a lifetime perspective. It is presented in conceptual terms in Figure 1. The underlying methodology is that of a Markov model. There are several mechanisms in the model that affect each other as illustrated in Figure 1, and each of them is described below. The model randomly assigns initial values to each patient, namely age, BMI, and gender. The patients' values are then updated annually until the model reaches the end of the covered time period. The control flow of the model is presented in Figure 2. Two patient groups with identical initial characteristics are simulated in this way, i.e. the surgery group and the control group. The surgery patients are assumed to have gastric bypass surgery. Patients in the comparator are given conventional non-surgical treatment.

\section{Figure 1: Conceptual model of a person eligible for gastric bypass surgery.}

Arrows indicate the influence of factors on patient characteristics. Surgery affects Body Mass Index (BMI), which together with age and gender affects life expectancy. Costs and Quality Adjusted Life years (QALY) are derived as a basis for the cost-effectiveness analysis.

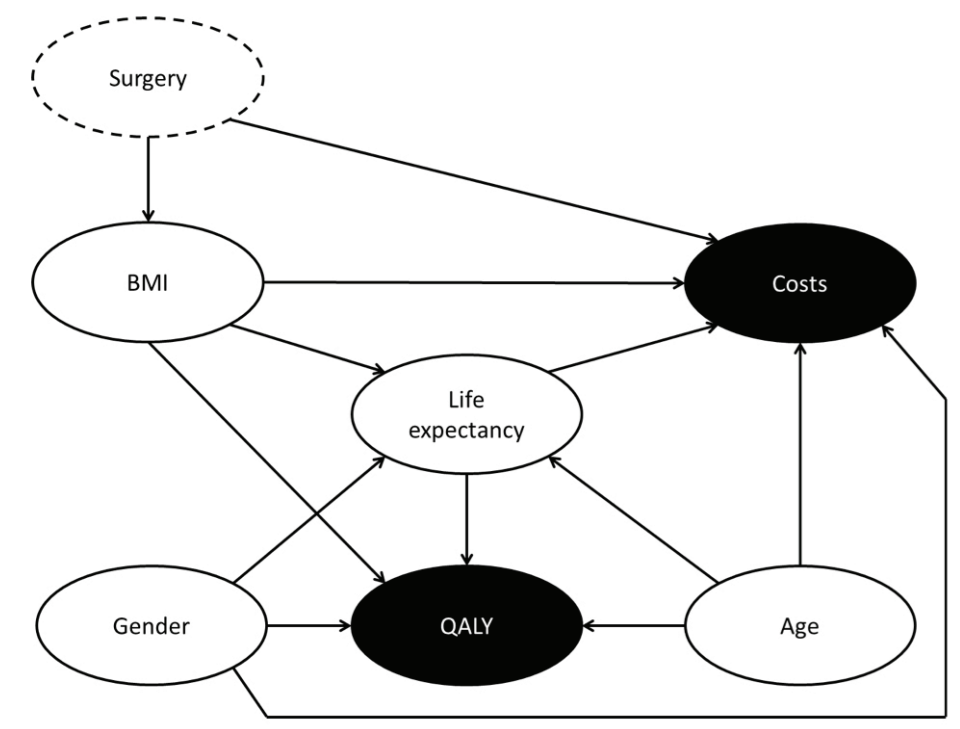


Figure 2: Control flow in the model

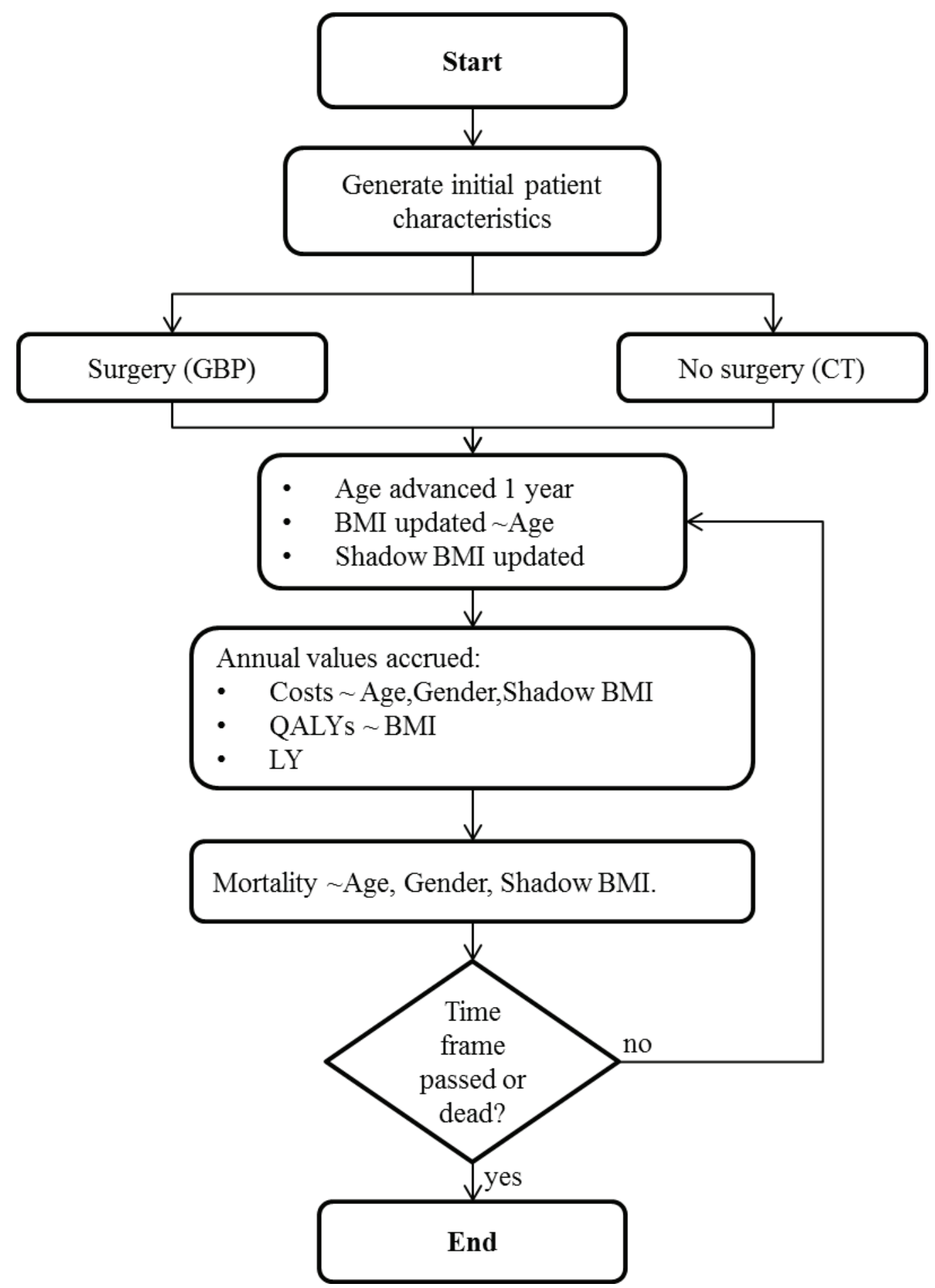

Notes: $\sim$ indicates dependency, e.g. QALY weights are dependent on BMI. For the definition of shadow BMI, see Section 2.2. GBP = Gastric Bypass surgery. $\mathrm{CT}=$ conventional treatment. LY $=$ Life years. The dependencies look different in some sensitivity analyses, e.g. QALYs depend on combinations of BMI, age and gender, mortality depend on BMI instead of Shadow BMI. Cost dependencies vary by type of cost. Surgery cost is accrued initially. 


\subsection{Surgery}

A patient given surgery will have a consequent change in BMI. However, adverse events may also occur. Sometimes plastic surgery is required to remove superfluous skin. Further, some patients experience small bowel obstruction sometime after surgery, which is usually treated surgically. Other less costly or less frequent adverse events can also occur, e.g. nutritional complications like iron deficiency, but we limited our adverse event analysis to small bowel obstruction.

\subsection{Surgery effectiveness}

The Swedish Obesity Study (SOS), a longitudinal intervention trial that has evaluated surgical treatment of obesity, reported that the average weight loss after the first year following gastric bypass surgery was 32 per cent (standard deviation 8\%), and after 15 years, 27\% (standard deviation 12\%), compared to the control group (Sjostrom 2013). The treatment in the control group was the actual non-surgical treatment given at the enrolled healthcare centres, ranging from sophisticated lifestyle intervention and behaviour modification to no treatment at all (Sjostrom 2013). Thus it appears that surgery achieves a weight reduction after one year, and that this weight reduction is to a great extent maintained over a long time. Since the BMI depends linearly on the patient's weight, we apply the estimated relative weight loss to the BMI. Conservatively, we assume that the effectiveness of the surgery in the model is a reduction of BMI by $27 \%$.

To avoid overestimating the cost and survival benefits from BMI reduction, the model determines a patient's mortality and excess costs using an adjusted BMI (henceforth denoted Shadow BMI). Whereas the BMI is reduced the first year after surgery, the Shadow BMI is reduced gradually over a ramp-up time of 3 years. Further, only $75 \%$ of the reduction (denoted maximum fraction of benefit) is applied to the Shadow BMI (Borg et al. 2012). Figure 3 illustrates the development of actual BMI and Shadow BMI over time, following surgery (denoted benefit of surgery), and without surgery. In two sensitivity analyses we explore a twice as high ramp-up time, and half the maximum fraction of benefit, to study the influence of these two parameters.

\subsection{Annual BMI increment}

Our model uses an annual increment to update a patient's BMI over time. The increment is $+0.12 \mathrm{~kg} / \mathrm{m}^{2}$ in patients aged $<45$ years, $+0.07 \mathrm{~kg} / \mathrm{m}^{2}$ for age 45 to 65 years, and -0.14 $\mathrm{kg} / \mathrm{m}^{2}$ for ages $\geq 65$ years, regardless of gender (Figure 3). Baum and Ruhm (2009) reported the increment for ages $<45$ years. The two other increments were estimated from the distribution of BMI in different age groups, and their relative risks of death by age group and BMI according to the NHANES studies (Flegal et al. 2005). The relative risks of death was hereby translated into mortality using the Swedish general population mortality as baseline (Statistics Sweden 2013b). We use these annual increments in both the surgery group and the control group, since we are not aware of any data pointing to the contrary.

\subsection{Mortality}

The mortality of the obese population is reported to be about twice as high as in the general population, for corresponding age and gender (Borg et al. 2005; Calle et al. 1999; Waaler 1984; Whitlock et al. 2009). We use the age and gender specific mortality of the Swedish general population in 2012 (Statistics Sweden 2013b). The mortality is accelerated using gender and BMI specific coefficients estimated from a recent analysis of some 900,000 adults (Whitlock et al. 2009), to account for excess mortality caused by 
Figure 3: Body Mass Index (BMI) over time

Change in BMI over time, depending on initial surgery or not, and the accrued benefit of surgery in the model.

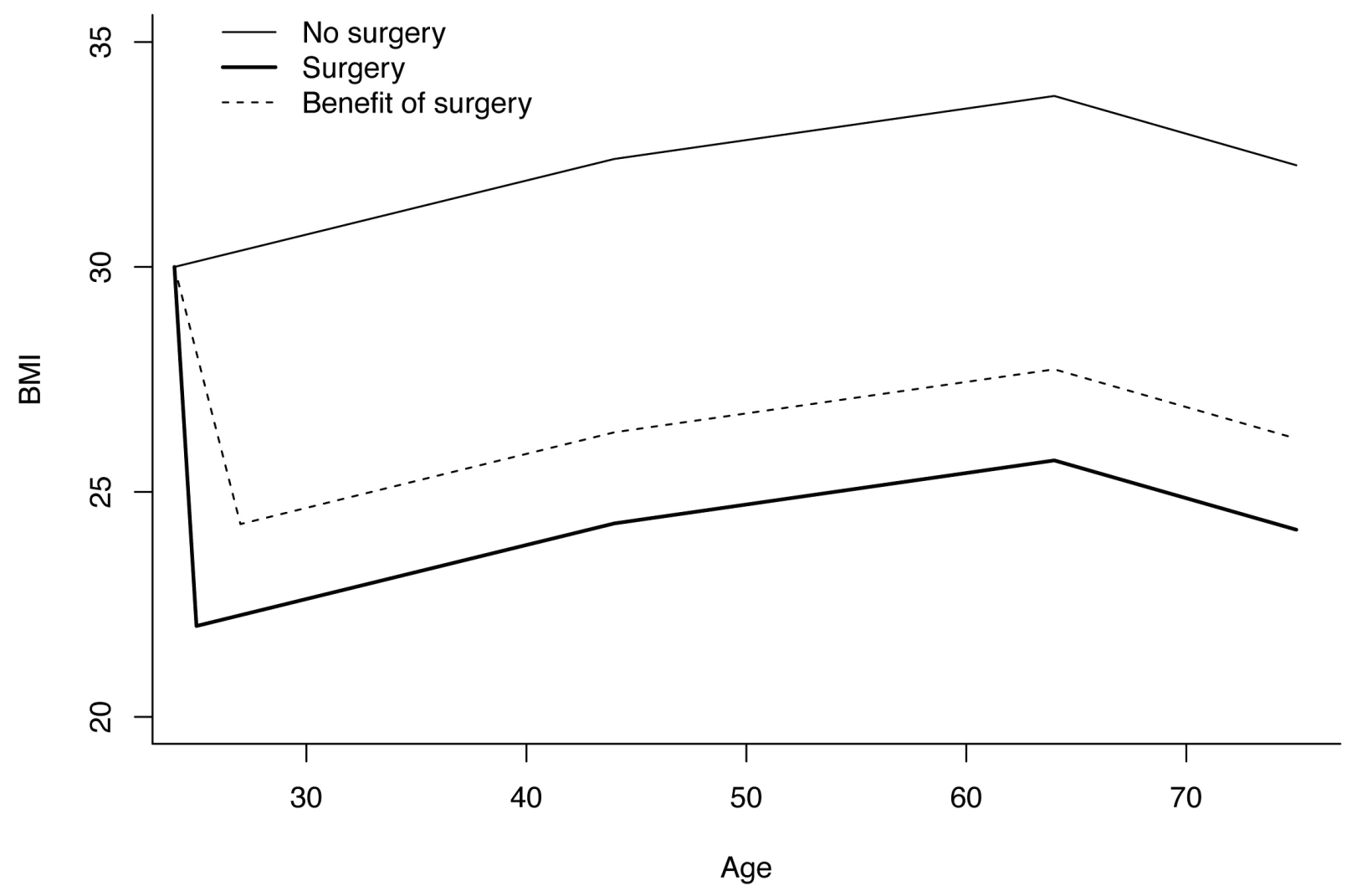

obesity. The relation between BMI and mortality is however surrounded by some uncertainty (see Discussion) (Angeras et al. 2013; Borg et al. 2005; Flegal et al. 2013; Whitlock et al. 2009), and therefore we also do a sensitivity with a mortality independent of BMI. This was accomplished by assigning everyone a mortality corresponding to a BMI of 42.5 , the midpoint of the base case interval.

Further, an obese patient undergoing surgery faces a risk of death in direct connection to the surgery. It has been reported from the SOS that 1 person died for every 500 surgeries performed (Sjostrom et al. 1995). We apply this mortality for patients undergoing surgery in the model.

\subsection{Costs}

Our model includes three types of direct costs, the intervention cost of the surgical procedure including any plastic surgery required, cost of adverse events, and excess healthcare costs of treating obesity related diseases. Furthermore it includes indirect costs of net consumption minus production, and productivity loss due to sick leave.

\subsubsection{Direct costs}

The surgical intervention cost includes the initial gastric bypass surgery, and any plastic surgery that may be needed afterwards. One of the major organizations performing gastric bypass surgery in Sweden, making about an eighth of all gastric bypass surgeries in Sweden, charges the healthcare system an amount per patient that depends on the ASA 
classification of severity of the patient (Dripps et al. 1961; Stoelting and Miller 1994). They receive SEK 52500 per ASA level 2 patient, and SEK 65000 to 70000 per ASA level 3 patient. Mild obesity falls into ASA level 2, and morbid obesity falls into ASA level 3. Probably, not all gastric bypass surgeries are carried out at this cost, e.g. a smaller clinic may not have the same economy of scale, and consequently has a higher cost. Nonetheless, based on morbid obesity usually being defined as $\geq 35 \mathrm{~kg} / \mathrm{m}^{2}$ in Europe, we assume that the intervention cost per patient is SEK 52500 if BMI $<35 \mathrm{~kg} / \mathrm{m}^{2}$, and SEK 67500 if $\mathrm{BMI} \geq 35 \mathrm{~kg} / \mathrm{m}^{2}$ (See discussion). According to e.g. the official hospital price lists in the Southern Healthcare Region in Sweden (Södra Regionvårdsnämnden 2011), a gastric bypass operation costs about SEK 95 000, however this reflect high risk patients. Another estimate of the cost of bariatric surgery is SEK 105400 (Lönroth et al. 2008), including SEK 90481 for surgery and the remainder for preoperative examinations and post-operative follow-up. In a sensitivity analysis, we therefore use a higher intervention cost of SEK 100000 regardless of BMI, reflecting roughly the mean of the latter two estimates.

To account for long-term adverse events, we added the expected cost of treatment for surgical treatment of small bowel obstruction to the intervention cost. The surgery is assumed to cost SEK 40100 per patient including 5 days of inpatient stay (Södra Regionvårdsnämnden 2011), and we assume that small bowel obstruction occur in $1.5 \%$ of the patients within one year from gastric bypass surgery, based on a study by Cho et al (2006).

The excess cost was defined as the difference in cost of treatment for obesity related diseases (diabetes, hypertension; cardiovascular disease; osteoarthritis and certain forms of cancer) between a normal weight patient and an overweight or obese patient. A key feature of our model is that the excess cost increases continuously with the BMI level. Since surgery reduces BMI, a reduction in excess treatment costs follows. We used estimates of excess obesity related healthcare costs per patients for different BMI and age groups (Borg et al. 2012), converted to the year 2012 price level (Table 1). We fitted functions $\mathrm{C}$ of BMI (x) to these excess costs estimates, namely $\mathrm{C}(\mathrm{x})=\mathrm{A}$ if $25 \leq \mathrm{x}<30$, and $\mathrm{C}(\mathrm{x})=\mathrm{A}+\mathrm{B}(\mathrm{x}-30)^{2}$ if $\mathrm{x} \geq 30$. By definition, the excess cost for a normal weight patient is zero. The coefficients $\mathrm{A}$ and $\mathrm{B}$ differ by gender and age, $\mathrm{A}$ is shown in Table 1 in the column for BMI 25-29, and B is 88.8 in male patients aged $<55$ years; 49.4 in female $<55$ years; 179.1 in male $\geq 55$ years; and 87.7 in female $\geq 55$ years.

Table 1: Excess treatment cost per capita related to overweight and obesity*, Sweden (Year 2012 SEK)

\begin{tabular}{lccccc}
\hline & \multicolumn{5}{c}{ BMI } \\
\hline Gender & Age & $\mathbf{2 5 - 2 9}$ & $\mathbf{3 0 - 3 4}$ & $\mathbf{3 5 - 3 9}$ & $\mathbf{4 0 -}$ \\
\hline \multirow{2}{*}{ Male } & $<\mathbf{5 5}$ years & 328 & 1,331 & 3,258 & 14,731 \\
& $\geq \mathbf{5 5}$ years & 715 & 2,690 & 6,588 & 29,560 \\
\hline \multirow{2}{*}{ Female } & $<\mathbf{5 5}$ years & 572 & 1,091 & 2,444 & 10,208 \\
& $\geq \mathbf{5 5}$ years & 1,251 & 1,966 & 2,194 & 18,400 \\
\hline
\end{tabular}

Notes: BMI = Body Mass Index.

* Excess costs compared to normal weight (BMI 18-25 kg/m²), from Borg et al (2012). 
In order to examine the influence of the functional form on the excess costs, we carried out a sensitivity analysis where the costs applied to the patients were exactly as shown in Table 1. In a follow-up study of the SOS patients, differences in healthcare resource consumption and costs over 20 years were presented (Neovius et al. 2012b). Assuming a cost per inpatient day of SEK 2818 and depending on how the presented drug cost estimates are adjusted to reflect 20 years of follow-up, the resulting difference in costs between a surgery patient and a control patient in their study falls in the range of SEK -1 100 to 8500 over twenty years, i.e. practically no difference. Therefore we also undertook a sensitivity analysis where we excluded the excess healthcare costs entirely.

\subsubsection{Indirect costs}

In order to value costs associated with extended life, we used a study by Ekman that estimated the difference between total annual consumption and total annual production per individual and by age (Ekman 2002). The difference is SEK 150317 (0-19 years of age), SEK -23 834 (20-34 years), SEK -129 184 (35-49 years), SEK -69 096 (50-64 years), SEK 153517 (65-74 years), SEK 186602 (75-84 years), and SEK 281196 (age 85 and above), per person in 2012 values. The estimated differences are not related to BMI and therefore it values extended life years regardless of BMI.

Neovius et al (2012) performed a study of young Swedish males and their sickleave during a follow-up period of up to 35 years, stratified by initial BMI. They presented incidence of sick leave by interval of sick-leave duration, $\leq 7$ days, 8-30 days and $>30$ days. We ascribed costs to these estimates using average salary including social fees (Ekonomifakta 2013; Statistics Sweden 2013c), at SEK 2224 per day of absence, and assumed durations of $3.5,15$, and 45 days respectively (Table 2). The resulting annual costs are related to initial BMI, not to longitudinally recorded BMI that would fit our modelling approach better. Nonetheless, we used it in a sensitivity analysis to get a rough estimate of productivity costs derived from a relationship with BMI. Note however, that since these BMI-related costs represent one term in the difference between consumption and production derived from Ekman, we do not use the Ekman data in this particular sensitivity analysis.

Table 2: Annual indirect costs of sick-leave (Year 2012 SEK), stratified by BMI in young adolescence (derived from Neovius et al (2012a).

\begin{tabular}{|c|c|c|c|c|c|}
\hline & \multicolumn{3}{|c|}{ Incidence per year } & \multirow{2}{*}{$\begin{array}{c}\text { Annual days of } \\
\text { absence* }\end{array}$} & \multirow{2}{*}{$\begin{array}{c}\text { Annual } \\
\text { Cost }\end{array}$} \\
\hline & long-term & intermediate & short term & & \\
\hline BMI 30.0- & 0.098 & 0.28 & 1.7 & 14.6 & 32381 \\
\hline BMI 25.0-29.9 & 0.083 & 0.24 & 1.5 & 12.6 & 27989 \\
\hline BMI 18.5-24.9 & 0.066 & 0.19 & 1.5 & 11.1 & 24620 \\
\hline BMI $<18.5$ & 0.063 & 0.19 & 1.7 & 11.6 & 25876 \\
\hline
\end{tabular}

Notes: * Assuming durations of 45, 15 and 3.5 days, respectively, for long-term ( $>30$ days), intermediate term (8-30 days) and short term sick-leave ( $\leq 7$ days). 


\subsection{QALYs and life years}

Each survived year in the model is recorded as a life year (LY), and weighted into a QALY estimate. We used QALY weights from the HODaR study, depending on the patient's BMI value (Ackroyd et al. 2006). We also undertook sensitivity analyses, where we used two sets of weights derived from bariatric surgery patients (Sauerland et al. 2009). Here, multiple regression models were used to obtain EQ-5D and SF-6D QALY weights depending on age, gender, BMI and bariatric surgery. In another sensitivity analysis we used the QALY weights for type 2 Diabetes patients reported from HODaR. In a further sensitivity analysis, we used BMI-dependent QALY weights from persons without chronic diseases in Finland (Korhonen et al. 2014).

\subsection{Patient characteristics and statistical analysis}

We explore age groups in ten year intervals, the youngest being 25-34 years and the oldest being 65-74 years of age, male and female patients, and BMI values ranging from 30 to 50 $\mathrm{kg} / \mathrm{m}^{2}$ in five unit intervals. We selected the stratum age 45-54 years and BMI 40-44 $\mathrm{kg} / \mathrm{m}^{2}$ as our base case. The model is evaluated using Monte Carlo simulation to estimate expected values given the parameter set. The number of patients was selected to make overall results stable across different random seeds. The costs, QALYs and LYs were discounted using a 3\% annual rate. The model was implemented in $\mathrm{R}$ (R Development Core Team 2009). All costs are presented in year 2012 SEK ( 1 SEK $\approx 0.11$ EUR $\approx 0.15$ USD).

\subsection{Sensitivity analyses}

To summarize, we carried out the following sensitivity analyses:

- Higher cost of the surgical intervention (SEK 100 000).

- Excess healthcare costs excluded.

- Step functions for excess costs instead of fitted polynomial functions. (Costs taken exactly as presented in Table 1).

- Use of BMI-related productivity costs due to absence from work instead of agerelated indirect costs of consumption minus production.

- Half the maximum fraction of benefit (37.5\%).

- Double ramp-up time (6 years).

- Mortality independent of BMI.

- Four alternative sets of QALY weights.

\section{Results}

In Table 3 we present the model's average outcomes per patient (costs, LY, and QALY) for gastric bypass surgery patients and conventional treatment patients, by initial BMI, age, and gender, in the base case. Compared to conventional treatment, surgery is estimated to gain QALYs in both men and women, to be cost saving in men and to be associated with increased costs in women with an incremental cost per QALY gained of SEK 26 thousand (Figure 4, Table 3). The costs are mainly indirect in the gastric bypass group, and excess healthcare costs in the conventional treatment group. Surgery costs are directly dependent on initial BMI, regardless of gender and age. Excess costs increase rapidly with initial BMI although less so in the surgery group, since surgery reduces BMI. When BMI is kept fixed (40-44) and age is varied, excess costs are driven by remaining life years, thus excess costs are higher in younger patients. BMI post-surgery is less (although initially 40-44), and therefore the excess costs are much lower. 


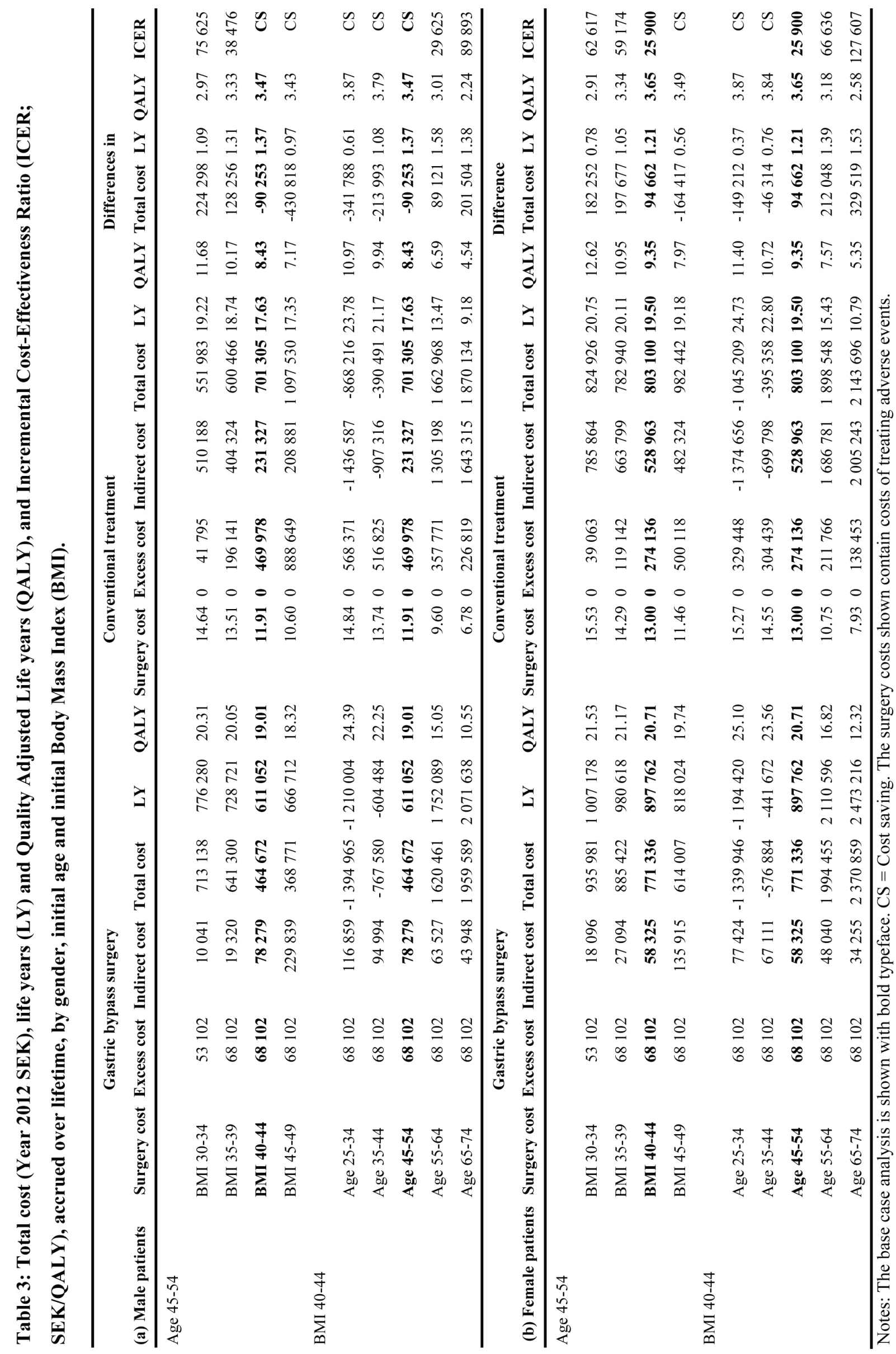


The pattern is similar in men and women. In the base case, the annual indirect costs depend only on age, thus when age is kept fixed (45-54), they are driven by remaining life years through gender specific mortality accelerated by BMI. When BMI is kept fixed (4044), initial age is driving indirect costs. Within the age range considered here, patients produce more than they consume up to the retirement age of 65 years, then they consume more than they produce. This is seen in the indirect costs. Surgery improves survival, which is seen in higher indirect costs in the surgery group. Remaining life years depend on age and initial BMI, and on surgery, which reduces BMI. The pattern is similar in men and women. The QALY estimates depend on the remaining life years and the patient's BMI over time, since the QALY weights in each time depend on the BMI.

\section{Figure 4: Costs, life years and QALYs}

Predicted costs of surgery (black), excess healthcare costs (grey) and indirect costs (dashed); and Life years (LY, black) and QALYs (dashed), per patient by gender (M/F) given Gastric Bypass surgery (GBP) or Conventional Treatment (CT). Costs presented in year 2012 SEK.
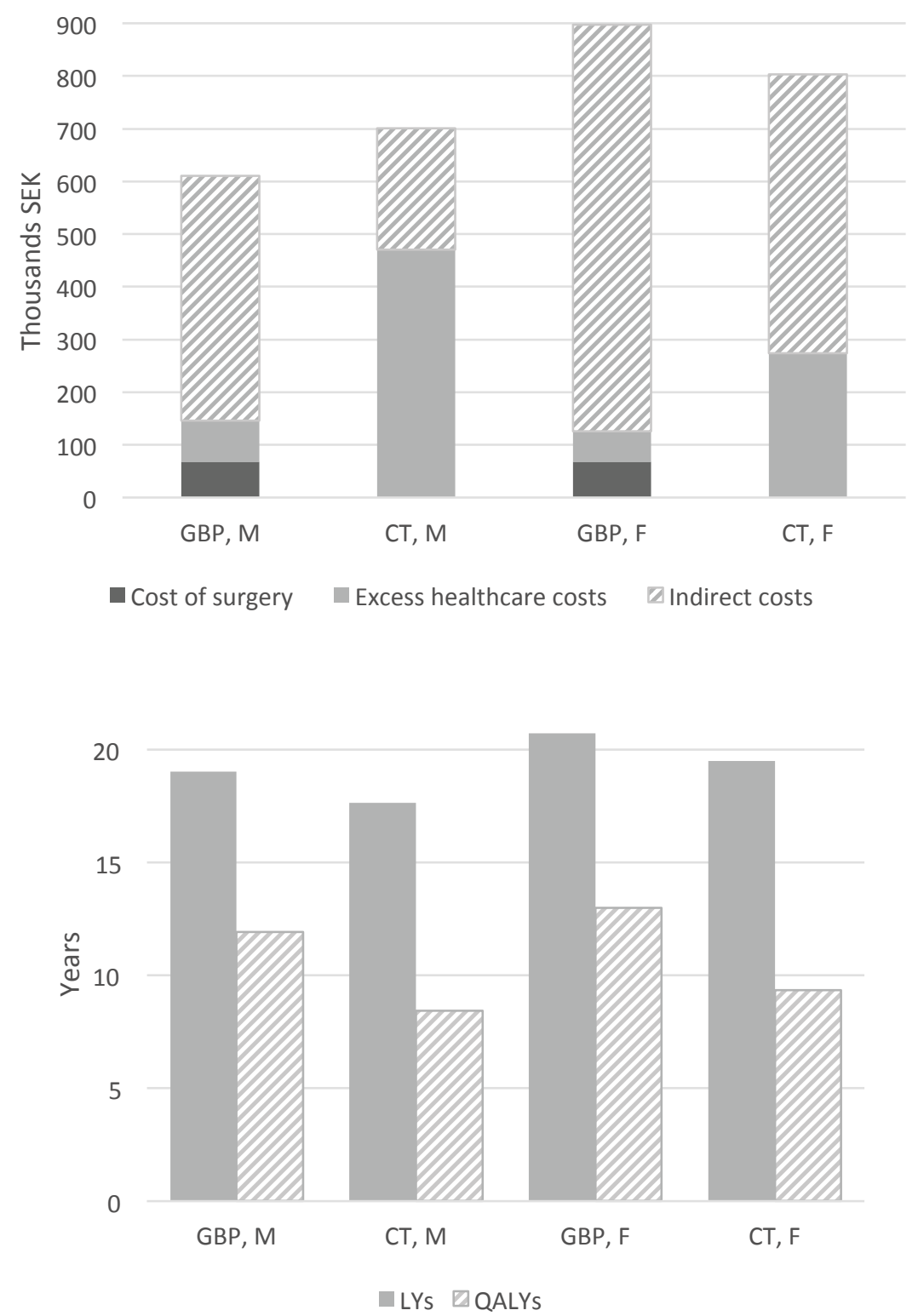

Note: Patients initially 45-54 years, with Body Mass Index (BMI) 40-44 kg/m². 
The results in the BMI strata in Table 3 indicate surgery is cost saving in men with BMI above 40 , and in women with BMI above 45 , but this also depends on their initial age. The younger the patient, the lower the cost in the surgery group since more production is offsetting the increased costs of consumption in extended life (Figure 5). The costs, the majority being indirect costs, are high on the absolute level, but similar between the gastric bypass group and the conventional treatment group. The incremental costs range between SEK -431 and 330 thousand, in the various age, gender and BMI strata (Table 3). Surgery is associated with an increase in life years and QALYs regardless of initial BMI, age, and gender. The incremental QALYs range between 2.24 and 3.87. Where surgery is not cost saving, the highest incremental cost per QALY gained (ICER) is seen in the oldest women, SEK 128 thousand per QALY gained.

Figure 5: Threshold for surgery being cost-saving

Threshold Body Mass Index (BMI) above which Gastric Bypass surgery is cost saving compared to conventional treatment.

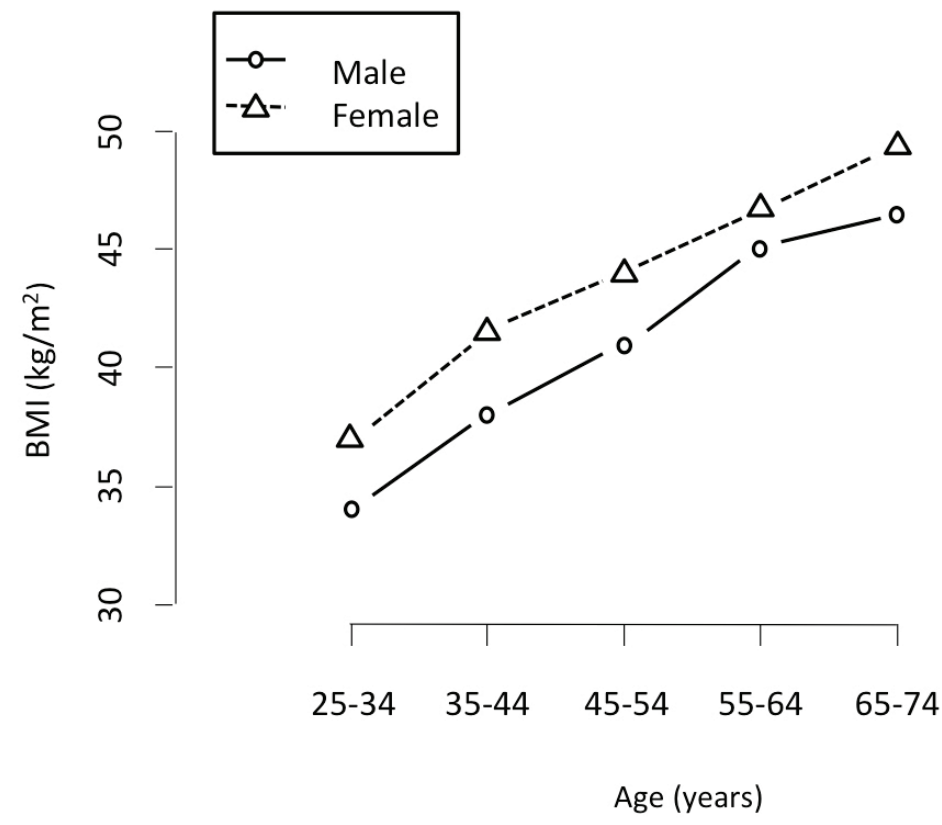

\subsection{Sensitivity analyses}

In the base case, surgery was associated with significant savings in excess costs. When excess costs were excluded from the analysis, surgery was instead associated with an incremental total cost of about SEK 300 thousand (both men and women), and the resulting ICER was about SEK 87 thousand per QALY gained, i.e. still within the range mentioned above. To examine the impact of using a polynomial function for the excess healthcare costs, we used a step function instead. This had a very minor effect, with the biggest change seen in women without surgery (Table 4). Our indirect costs depend only on age in the base case, given remaining life years, which in turn depend on BMI and gender. We used alternative indirect costs that only depend on BMI in a sensitivity analysis. This had significant impact on the indirect costs, which were increased except in women with surgery, and carried over to total costs, however to the advantage of surgery in both men and women (Table 4). With a higher surgical intervention cost, surgery is still cost saving in men. In women, the incremental cost becomes larger, resulting in an ICER of SEK 35 thousand per QALY gained. 


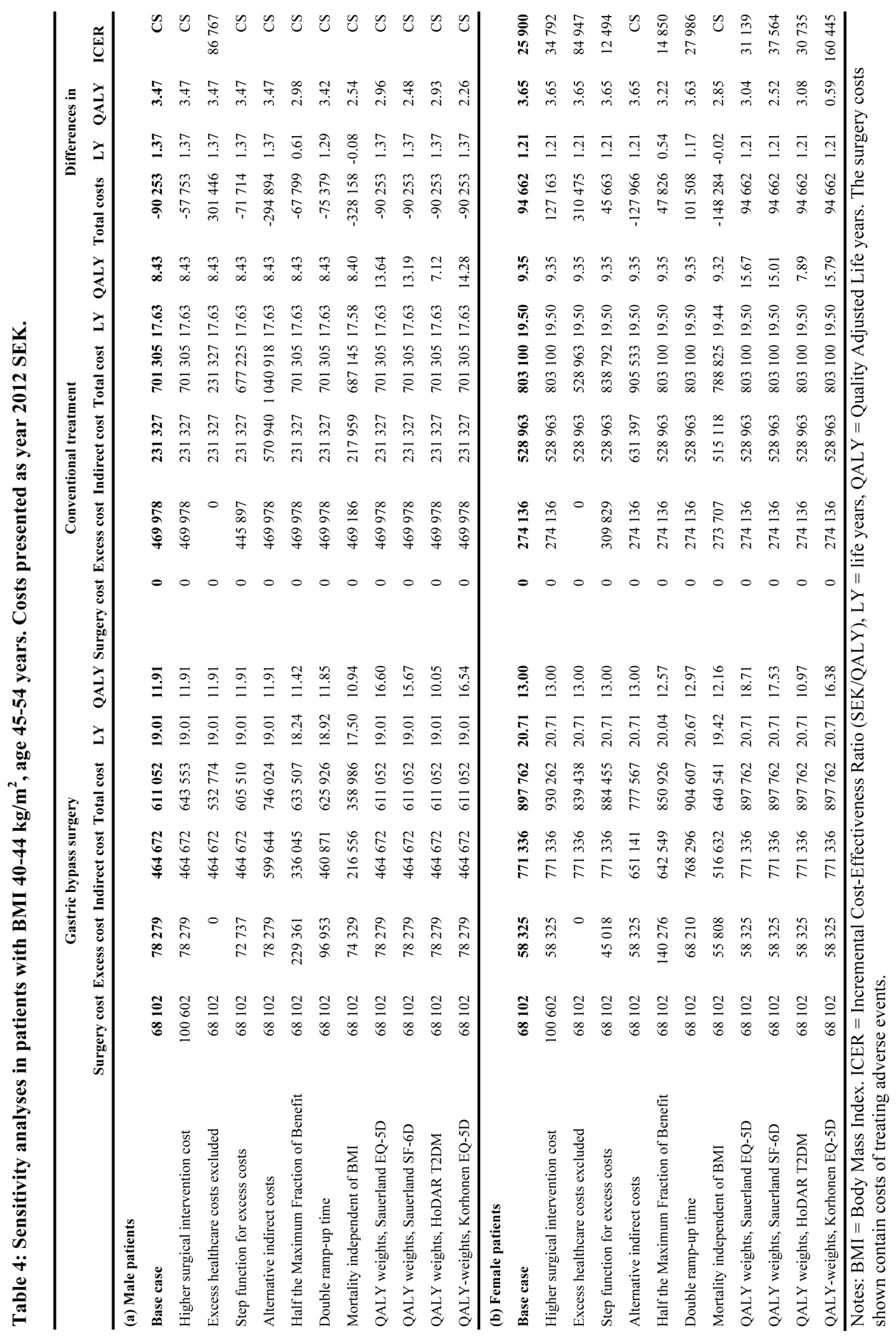


Two sensitivity analyses were carried out to investigate the impact of how the effectiveness of surgery is modelled. Using a double ramp-up time, i.e. that the full effectiveness of surgery would be reached after twice the amount of time, increased the excess costs somewhat and slightly decreased the QALYs gained (Table 4). Using only half the maximum fraction of benefit compared to the base case, resulted in a large increase in excess costs, a decrease in indirect costs and a decrease in QALYs gained.

In a sensitivity analysis where the mortality did not depend on BMI, the QALY gain depends only on QALY weights being higher, not on any gain in life years (apart from noise). Without any benefit in survival, the indirect costs in the gastric bypass group is about half those in the base case, whereas it is unchanged in the conventional treatment group. There is a difference of about SEK 13 thousand compared to the base case in both groups which depends on how the mortality was made independent of BMI (Section 2.4). In both men and women gastric bypass is cost saving compared to conventional treatment, because of the avoided indirect costs in the gastric bypass group.

In our sensitivity analyses with alternative QALY weights, the weights from Korhonen et al gave the biggest change in QALY difference between gastric bypass and conventional treatment. The estimated QALY differences in women ranges from 0.59 to 3.65 (Table 4), and the ICER ranges from SEK 30 to 160 thousand per QALY gained. In men, the QALY difference ranges from 2.26 to 2.96 , and surgery is estimated to be cost saving, i.e. regardless of the QALY difference.

\section{Discussion}

We have studied the cost-effectiveness of gastric bypass surgery compared to conventional treatment in a modelling study. Depending on initial patient characteristics, surgery is estimated to be either cost saving or associated with an incremental cost per QALY gained not above SEK 128 thousand. Although there is no official threshold of an incremental cost per QALY gained for an intervention to be judged acceptable in a Swedish context, we believe that the incremental costs per QALY gained that we have found are modest, and that surgery should be regarded as cost-effective compared to conventional treatment.

We investigated how assumptions regarding surgery effectiveness would affect the analysis. Overall, the incremental analysis was fairly robust to these changes. In SOS, it was seen that the reversibility rate of BMI following surgery differed across different comorbidities (Sjostrom et al. 2007). Efficacy in terms of morbidity reduction was also observed to differ depending upon presence of co-morbidity or not. We have not been able to assess how such differences in reversibility rate may bias our model results.

Flegal et al (2013) studied all-cause mortality and found lower mortality in overweight than in normal weight subjects, but higher mortality in obese subjects than in normal weight subjects. This differs from our hypothesis with a mortality that increases with BMI. Angerås et al (2013) found a pattern of all-cause mortality in patients with acute coronary syndromes which was similar to the findings of Flegal et al. This is in contrast to our own study of some 33000 Swedish subjects, where we detected no difference in mortality between normal weight and overweight (Borg et al. 2005). Nor was there any numerical indication of a lower mortality in overweight compared to normal weight. Further, Nilsson et al (2002) studied non-cancer mortality in a male Swedish population experiencing weight loss. They found a higher mortality in subjects experiencing weight loss than in subjects with stable weight. They raise the suspicion that this could be due to premature ageing effects causing so-called weight loss of involution. We do not see that this should necessarily translate into our situation with weight loss for 
a specific reason known in advance, namely gastric bypass surgery. We nonetheless find that uncertainty surrounds the relation between BMI and mortality. Our model links mortality to the adjusted BMI (Shadow BMI), not to the fully reduced BMI, and this should reasonably lessen any overestimation of the mortality reduction. However, this does not address a similar mortality in normal weight and overweight patients, nor a reverse relation between them. Therefore we carried out a sensitivity analysis using mortality independent on BMI, i.e. it eliminates any survival benefit of surgery. This resulted in surgery being highly cost saving in both men and women. Thus it would appear like our modelling of survival in the base case does not exaggerate the cost-effectiveness of gastric bypass. The choice of QALY weights has a clear impact on the QALY estimates on the absolute level, but it has little impact in the incremental analysis. This is mainly because the incremental costs are modest, and this prevents the incremental costeffectiveness ratios from becoming particularly high when the difference in QALYs varies.

We have probably underestimated the intervention cost by basing our assumption on one of the largest operators on the market. However, as this operator charge the healthcare system the prices we use, it must be possible to carry out the operations at that cost. Thus, this operator is technically efficient and, although not all gastric bypass surgeries carried out are performed by technically efficient operators, this would be theoretically possible. Still, we carried out a sensitivity analysis with a higher surgery cost, and gastric bypass surgery appears to remains cost-effective. Further sensitivity analyses were made regarding excess healthcare costs. The way excess costs increase by BMI may seem implausible for very high BMI values, but this has little impact since the prevalence of very high BMI values is low. Nevertheless, we examined alternative ways for how excess costs are modelled (and also to exclude them entirely), and our results remain robust. They appear to be robust for the choice of indirect costs as well.

The development of BMI over time is associated with uncertainty as well. Lacking other data, we assume the same annual BMI increments in surgery patients as in patients given non-surgery treatment. We have not examined alternative ways for BMI to change over time. However, our sensitivity analyses of worse surgery effectiveness, our sensitivity analysis of BMI independent mortality, and our sensitivity analysis without excess costs, and those with different QALY weights, could be regarded as proxies for alternative ways for BMI to develop over time, by looking at the values derived from the BMI. Our results appear robust to all these changes.

Our approach to assessing uncertainty has been to perform deterministic sensitivity analyses. The aim of this was to understand the drivers of the results, by modifying different parameters within plausible ranges, one parameter at a time. This type of analysis aims to help evaluate, for example, how the cost components or resources influence the result, and how strong that influence might be. However, a stochastic evaluation of the model would consider the uncertainty in all input values, each according to the observed variation and with the uncertainty in all components taken into account simultaneously. This would allow an evaluation of the overall uncertainty. Due to incomplete data for this approach, and our judgement that assumed distributions would not adequately compensate for the lack of data, we did not perform this kind of analysis, which is a weakness in our study.

Our findings are in line with finding from other studies reported in the literature. In a modelling study by Ackroyd et al (2006), it is reported on the cost-effectiveness of two surgical treatments, adjustable gastric banding and gastric bypass surgery, for obesity in adult patients with type 2 Diabetes compared with conventional treatment. The authors also conducted a review of BMI reduction associated with gastric bypass. The results 
show that the BMI reduction was $17.7 \mathrm{~kg} / \mathrm{m}^{2}$ in the first year, and 16 to $17 \mathrm{~kg} / \mathrm{m}^{2}$ annually in the subsequent 4 years. The authors presented three country-specific applications. Both in Germany and in France, the extra cost of surgery for gastric bypass was more than offset by a reduction in health care costs associated with type 2 Diabetes. In the UK, the incremental cost per QALY gained with gastric bypass over conventional treatment was $£ 1,517$. In a case-control study to evaluate the third-party payer return on investment in bariatric surgery in the US, an employer claims database was used to identify 3651 morbid obese patients (BMI 40+) who underwent bariatric surgery (Cremieux et al. 2008). They were matched to a group of control subjects who never underwent bariatric surgery. The results showed that all costs associated with the bariatric surgery investment had been recouped within 2 years for laparoscopic surgery patients and within 4 years for open surgery patients. A modelling study by Faria et al (2013), compared gastric bypass with best medical treatment in Portugal over lifetime. Assuming their best medical treatment and our conventional treatment are comparable, their overall picture matches ours although costs are difficult to translate between countries. They found gastric bypass to render 1.9 extra QALYs and to save Euro 13244 of healthcare costs (about SEK 120 thousand). Our estimate of the QALY gain is 0.6 to 3.7 depending on instrument used and the patient's gender, and we estimate the incremental healthcare cost of SEK -390 thousand in men and SEK -216 thousand in women. Both the study by Faria et al and our own found that surgery is more cost-effective in young patients and in patients with higher BMI. A study in Finland by Mäklin et al (2011) found surgery to be cost-saving and associated with a QALY gain of 0.6, which is lower than our base case estimate. However, they used a much shorter time horizon of only ten years during which they accumulated QALYs. On the other hand, we come closer to Mäklin's estimate when we use Korhonen's Finnish QALY weights, about 2.3 gained QALYs in men and 0.6 in women.

In summary we believe that the weaknesses of the cost-effectiveness models reviewed by Padwal et al (2011) highlight the strength of our model in that the efficacy of surgery in our model is based on long term observational data (15 years), that our model accommodates an age dependent up-drift and down-drift of weight, and that the effect on quality of life and reduced treatment costs of obesity related diseases are accommodated. Furthermore, our results appear to agree with those in other studies in so far that costs can be approximately compared between countries. So it appears that our initial question, whether it would be worthwhile to carry out gastric bypass surgery, has been positively answered. This does not mean that other measures may not be worthwhile too, like prevention of overweight and obesity.

\section{Conclusions}

According to our model, gastric bypass surgery appears to be cost-effective compared to conventional treatment. The incremental cost associated with gastric bypass decreases with BMI, increases with the patient's age, and is higher in women than in men. The incremental cost per QALY gained is estimated not to exceed SEK 90 thousand in men, and SEK 128 thousand in women (about EUR 10 and 14 thousand, respectively). Taking account of uncertainty in input data and model design, the incremental cost is estimated to at most SEK 160 thousand per QALY gained (about EUR 18 thousand per QALY). 


\section{References}

Ackroyd, R., Mouiel, J., Chevallier, J. M., and Daoud, F. (2006). Cost-effectiveness and budget impact of obesity surgery in patients with type-2 diabetes in three European countries. Obesity Surgery, 16(11), 1488-503.

Angeras, O., Albertsson, P., Karason, K., Ramunddal, T., Matejka, G., James, S., Lagerqvist, B., Rosengren, A., and Omerovic, E. (2013). Evidence for obesity paradox in patients with acute coronary syndromes: a report from the Swedish Coronary Angiography and Angioplasty Registry. European Heart Journal, 34(5), 345-53.

Avenell, A., Broom, J., Brown, T. J., Poobalan, A., Aucott, L., Stearns, S. C., Smith, W. C., Jung, R. T., Campbell, M. K., and Grant, A. M. (2004). Systematic review of the long-term effects and economic consequences of treatments for obesity and implications for health improvement. Health Technology Assessment, 8(21), iii-iv, 1-182.

Baum, C. L., 2nd, and Ruhm, C. J. (2009). Age, socioeconomic status and obesity growth. Journal of Health Economics, 28(3), 635-48.

Borg, S., Naslund, I., Persson, U., and Odegaard, K. (2012). Budget impact analysis of surgical treatment for obesity in Sweden. Scandinavian Journal of Surgery: SJS: official organ for the Finnish Surgical Society and the Scandinavian Surgical Society, 101(3), 190-7.

Borg, S., Persson, U., Ödegaard, K., Berglund, G., Nilsson, J. Å., and Nilsson, P. M. (2005). Obesity, survival, and hospital costs - Findings from a screening project in Sweden. Value in Health, 8(5), 562-571.

Calle, E. E., Thun, M. J., Petrelli, J. M., Rodriguez, C., and Heath, C. W., Jr. (1999). Body-mass index and mortality in a prospective cohort of U.S. adults. New England Journal of Medicine, 341(15), 1097-105.

Cho, M., Carrodeguas, L., Pinto, D., Lascano, C., Soto, F., Whipple, O., Gordon, R., Simpfendorfer, C., Gonzalvo, J. P., Szomstein, S., and Rosenthal, R. J. (2006). Diagnosis and management of partial small bowel obstruction after laparoscopic antecolic antegastric Rouxen-Y gastric bypass for morbid obesity. Journal of the American College of Surgeons, 202(2), $262-8$.

Chua, T. Y., and Mendiola, R. M. (1995). Laparoscopic Vertical Banded Gastroplasty: The Milwaukee Experience. Obesity Surgery, 5(1), 77-80.

Clegg, A. J., Colquitt, J., Sidhu, M. K., Royle, P., Loveman, E., and Walker, A. (2002). The clinical effectiveness and cost-effectiveness of surgery for people with morbid obesity: a systematic review and economic evaluation. Health Technology Assessment, 6(12), 1-153.

Cremieux, P. Y., Buchwald, H., Shikora, S. A., Ghosh, A., Yang, H. E., and Buessing, M. (2008). A study on the economic impact of bariatric surgery. American Journal of Managed Care, 14(9), 589-96.

Dripps, R. D., Lamont, A., and Eckenhoff, J. E. (1961). The role of anesthesia in surgical mortality. JAMA: The Journal of the American Medical Association, 178, 261-6.

Ekman, M. (2002). Consumption and production by age in Sweden: Basic facts and health economic implications. In Ekman, M., Studies in health economics: Modelling and data analysis of costs and survival. Dissertation for the degree of Doctor of Philosophy at the Stockholm School of Economics. EFI, Stockholm Sweden.

Ekonomifakta. (2013). Facts and statistics about Sweden's economy (www.ekonomifakta.se). Stockholm. 
Faria, G. R., Preto, J. R., and Costa-Maia, J. (2013). Gastric bypass is a cost-saving procedure: results from a comprehensive Markov model. Obesity Surgery, 23(4), 460-6.

Finkelstein, E. A., Fiebelkorn, I. C., and Wang, G. (2003). National medical spending attributable to overweight and obesity: how much, and who's paying? Health Affairs (Millwood), Suppl Web Exclusives, W3-219-26.

Flegal, K. M., Graubard, B. I., Williamson, D. F., and Gail, M. H. (2005). Excess deaths associated with underweight, overweight, and obesity. JAMA: The Journal of the American Medical Association, 293(15), 1861-7.

Flegal, K. M., Kit, B. K., Orpana, H., and Graubard, B. I. (2013). Association of all-cause mortality with overweight and obesity using standard body mass index categories: a systematic review and meta-analysis. JAMA: The Journal of the American Medical Association, 309(1), $71-82$.

Korhonen, P. E., Seppala, T., Jarvenpaa, S., and Kautiainen, H. (2014). Body mass index and health-related quality of life in apparently healthy individuals. Quality of life research: an international journal of quality of life aspects of treatment, care and rehabilitation, 23(1), 6774.

Lönroth, H., Stenlöf, K., Fändriks, L., and Olbers, T. (2008). Mini-HTA. Obesitaskirurgi [Miniature HTA Obesity surgery]. Online: www.sahlgrenska.se, Accessed 10 March 2014, Sahlgrenska universitetssjukhuset, Gothenburg, Sweden.

Maklin, S., Malmivaara, A., Linna, M., Victorzon, M., Koivukangas, V., and Sintonen, H. (2011). Cost-utility of bariatric surgery for morbid obesity in Finland. The British Journal of Surgery, 98(10), 1422-9.

Martin, L. F., Tan, T. L., Horn, J. R., Bixler, E. O., Kauffman, G. L., Becker, D. A., and Hunter, S. M. (1995). Comparison of the costs associated with medical and surgical treatment of obesity. Surgery, 118(4), 599-606; discussion 606-7.

Neovius, K., Neovius, M., Kark, M., and Rasmussen, F. (2012a). Association between obesity status and sick-leave in Swedish men: nationwide cohort study. European Journal of Public Health, 22(1), 112-6.

Neovius, M., Narbro, K., Keating, C., Peltonen, M., Sjoholm, K., Agren, G., Sjostrom, L., and Carlsson, L. (2012b). Health care use during 20 years following bariatric surgery. JAMA: The Journal of the American Medical Association, 308(11), 1132-41.

Nguyen, N. T., Goldman, C., Rosenquist, C. J., Arango, A., Cole, C. J., Lee, S. J., and Wolfe, B. M. (2001). Laparoscopic versus open gastric bypass: a randomized study of outcomes, quality of life, and costs. Annals of Surgery, 234(3), 279-89; discussion 289-91.

Nilsson, P. M., Nilsson, J. A., Hedblad, B., Berglund, G., and Lindgarde, F. (2002). The enigma of increased non-cancer mortality after weight loss in healthy men who are overweight or obese. Journal of Internal Medicine, 252(1), 70-8.

Padwal, R., Klarenbach, S., Wiebe, N., Hazel, M., Birch, D., Karmali, S., Sharma, A. M., Manns, B., and Tonelli, M. (2011). Bariatric surgery: a systematic review of the clinical and economic evidence. Journal of General Internal Medicine, 26(10), 1183-94.

Persson, U., and Ödegaard, K. (2011). Fetma, ett ekonomiskt samhällsproblem - kostnader och möjliga åtgärder för Sverige. Ekonomisk debatt, 2011(1).

R Development Core Team. (2009). R: A language and Environment for Statistical Computing. The R Foundation for Statistical Computing, Vienna, Austria. 
Sauerland, S., Weiner, S., Dolezalova, K., Angrisani, L., Noguera, C. M., Garcia-Caballero, M., Rupprecht, F., and Immenroth, M. (2009). Mapping utility scores from a disease-specific quality-of-life measure in bariatric surgery patients. Value in Health, 12(2), 364-70.

SBU. (2002). Fetma - problem och åtgärder. En systematisk litteraturöversikt (Obesity - problems and measures. A litterature review). Statens beredning för medicinsk utvärdering (SBU), Stockholm.

Scandinavian Obesity Surgery Registry. (2012). Annual Report 2012, part 1. Scandinavian Obesity Surgery Registry, Universitetssjukhuset, Örebro.

Sjostrom, L. (2013). Review of the key results from the Swedish Obese Subjects (SOS) trial - a prospective controlled intervention study of bariatric surgery. Journal of Internal Medicine, 273(3), 219-34.

Sjostrom, L., Narbro, K., Sjostrom, C. D., Karason, K., Larsson, B., Wedel, H., Lystig, T., Sullivan, M., Bouchard, C., Carlsson, B., Bengtsson, C., Dahlgren, S., Gummesson, A., Jacobson, P., Karlsson, J., Lindroos, A. K., Lonroth, H., Naslund, I., Olbers, T., Stenlof, K., Torgerson, J., Agren, G., and Carlsson, L. M. (2007). Effects of bariatric surgery on mortality in Swedish obese subjects. New England Journal of Medicine, 357(8), 741-52.

Sjostrom, L., Narbro, K., and Sjostrom, D. (1995). Costs and benefits when treating obesity. International Journal of Obesity and Related Metabolic Disorders, 19 Suppl 6, S9-12.

Statistics Sweden. (2013a). Undersökningarna av levnadsförhållandena (ULF/SILC). Statistics Sweden (www.scb.se), Stockholm.

Statistics Sweden. (2013b). Population statistics. Statistics Sweden (www.scb.se), Stockholm.

Statistics Sweden. (2013c). Labour market statistics. Statistics Sweden (www.scb.se), Stockholm.

Stoelting, R. K., and Miller, R. D. (1994). Basics of anesthesia, New York: Churchill Livingstone.

Södra Regionvårdsnämnden. (2011). Regionala priser och ersättningar för Södra sjukvårdsregionen 2012. Online: www.skane.se, Accessed 4 June 2013.

Waaler, H. T. (1984). Height, weight and mortality. The Norwegian experience. Acta Medica Scandinavica Supplementum, 679, 1-56.

van Gemert, W. G., Adang, E. M., Kop, M., Vos, G., Greve, J. W., and Soeters, P. B. (1999). A prospective cost-effectiveness analysis of vertical banded gastroplasty for the treatment of morbid obesity. Obesity Surgery, 9(5), 484-91.

Whitlock, G., Lewington, S., Sherliker, P., Clarke, R., Emberson, J., Halsey, J., Qizilbash, N., Collins, R., and Peto, R. (2009). Body-mass index and cause-specific mortality in 900000 adults: collaborative analyses of 57 prospective studies. Lancet, 373(9669), 1083-96.

WHO. (2005). The World Health Organization warns of the rising threat of heart disease and stroke as overweight and obesity rapidly increase. The World Health Organization, Geneva. Online, www.who.int, Accessed 14 March 2014.

WHO. (2013). Obesity and overweight. The World Health Organization, Geneva. On line, www.who.int, Accessed 14 March 2014.

WHO/NUT/NCD/98. (1997). Obesity. Preventing and managing the global epidemic. Report of WHO Concultation on Obesity, Geneva, 3-5 june 1997. 